\title{
Pedestrian Safety - Exploring the Different Applications for Pedestrian Sensing Detection
}

\author{
Satish Ullatil \\ Master of Technology - Automotive Engineering \\ Second Semester Student, WIPL, Birla Institute of Technology \& Science, Pilani \\ Benjamin Surowiec, Innovation Project Manager (Mentor): Mechanics Group Co-coordinator, \\ Plastic Omnium Intelligent Exteriors Systems, Sigmatech, Sainte Julie, France
}

\begin{abstract}
According to the National Highway Traffic Safety Administration about 5,400 pedestrians were killed and over 70,000 were injured in traffic accidents in 2015 . On average, a pedestrian was killed every 1.6 hours and injured every 7.5 minutes in the U.S. that year. Clearly, pedestrians are in danger, and drivers need to do more to protect them. Car manufacturers have stepped up to help by offering systems that detect pedestrians and bicyclists and warn the driver.
\end{abstract}

Key Words: Camera based pedestrian protection, Sensing Principle, Early crash sensors, Active Sensor, LIDAR, Camera, Radar

\section{INTRODUCTION}

Pedestrians are particularly at risk in traffic. In most accidents, the pedestrian collides with the front of the vehicle The requirement of Pedestrian detection is an essential and significant task in any intelligent video surveillance system, as it provides the fundamental information for semantic understanding of the video footage. It has an obvious extension to automotive applications due to the potential for improving safety systems. Product OEM's have developed an electronic system for active impact protection for pedestrians which is a safe and cost-effective solution and also fulfil the legal requirements for pedestrian protection on vehicles and predictive pedestrian protection system which can detect impending accidents with pedestrians who are in the same lane as the vehicle or who are moving dangerously into this direction.

\section{OBJECTIVE}

The objective of this paper is to address the different approaches and methods to reduce the pedestrian fatal injuries or accidents by integrating the safety feature on vehicle front end or as separate unit assembled to the vehicle and using advanced sensors to detect human movement on autonomous vehicles.

\section{PEDESTRIAN SAFETY METHODS}

There are few safety methods being introduced by leading OEM manufacture's such as Continental [1] and Bosch [2]

1. Intelligent Crash sensor.

2. Pressure based system.

3. Pedestrian Sensing \& Detection.

4. Video based approach.

5. Active Sensor approach.
6. Radar based approach.

7. Laser.

8. Lidar.

\section{1: Intelligent Crash Sensor}

The intelligent crash sensors are complement to the speed airbag control unit in a crash. They are based on robust technology and acceleration satellites [3]. The compact sensors are concealed in the bumper which sends a signal to the speed control unit when a collision occurs. The control unit can then raise the hood in a matter of milliseconds, creating a deeper crumple zone that prevents the pedestrian's head from hitting the engine block and reduces the fatal injury. The acceleration-based pedestrian protection system can enhanced by combining with pressure-based technology.

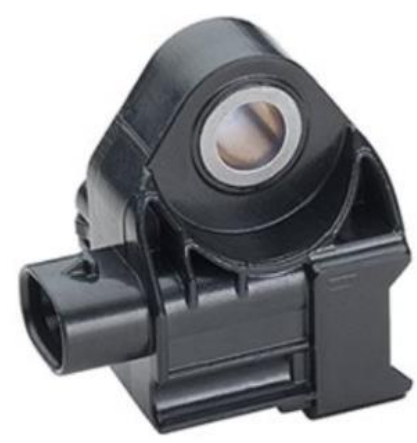

Fig1: Crash Sensor

The sensor provides protection for frontal and side impacts as well as from pedestrian protection which supply information on the acceleration values in the rigid vehicle structures in the event of a crash. They are used in conjunction with the pressure satellite, acceleration satellites offer an optimum solution for the rapid discrimination of side impacts. When the two technologies interact with each other, a synergetic value is obtained. In a crash, the pSAT [3]immediately senses any pressure change in the door, and the gSAT [4] then measures the lateral acceleration. With this fast, precise information, the airbag control unit can activate the side air bags in just a few milliseconds. 


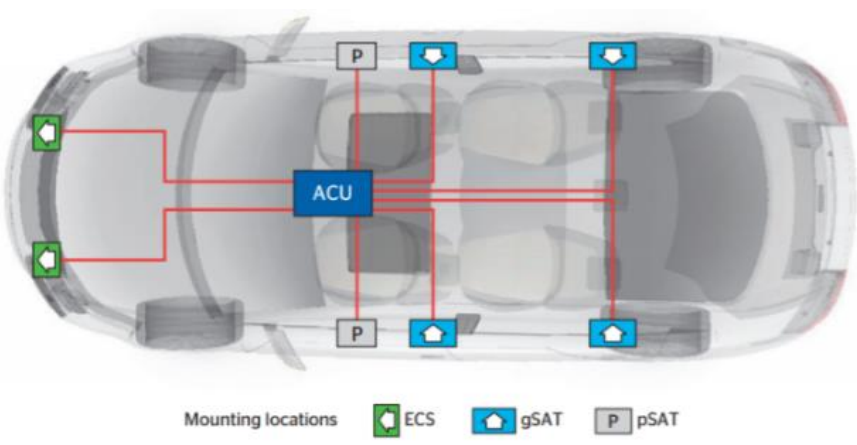

Fig2: Sensor Mounting Locations

\section{2: Pressure Based System}

The pedestrian protection sensor consists of a flexible air tube that is laid across the entire width of the car in its front bumper.

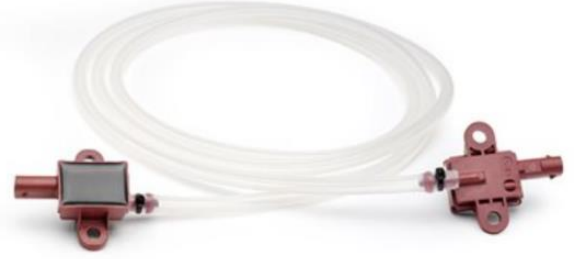

Fig3: Pressure Based System

The pedestrian protection sensor consists of a flexible air tube that is laid across the entire width of the car in its front bumper. The tube is situated directly behind the foam block that is fitted at the front of the vehicle to absorb impact energy. Standardized pressure sensors (pSAT) [3] are installed at either end of the air-filled pressure tube. When a vehicle collides with an obstacle, the resulting pressure exerted on the tube through the front bumper and foam block creates a typical waveform that is detected by the two sensors at the ends of the tube and forwarded to the speed airbag control unit.

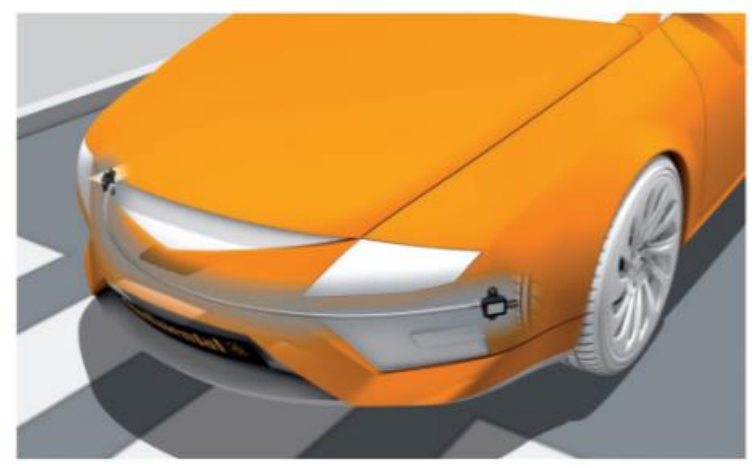

Fig3: Sensor Mounting Locations

Example on above image the front right-hand corner or the middle of the vehicle. Within 10-15 milliseconds of an impact, the active hood of the vehicle is triggered and raised by special actuators. This reduces the risk of death or severe injury to the pedestrian from hitting the hood and underlying engine block. The sensor is easily adaptable to any vehicle type. The pressure-based pedestrian protection system can be enhanced by combining with acceleration-based technology (gSAT).

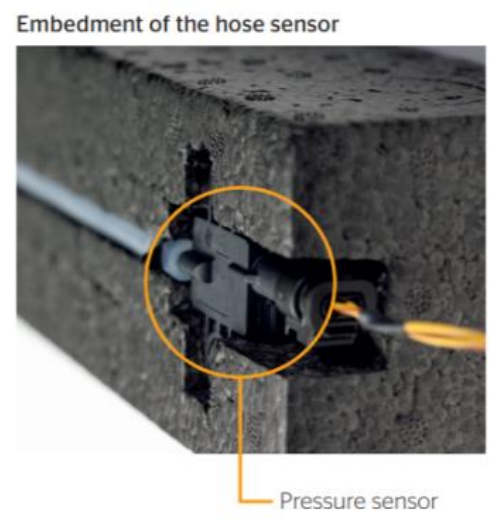

Fig4: Pressure sensor location

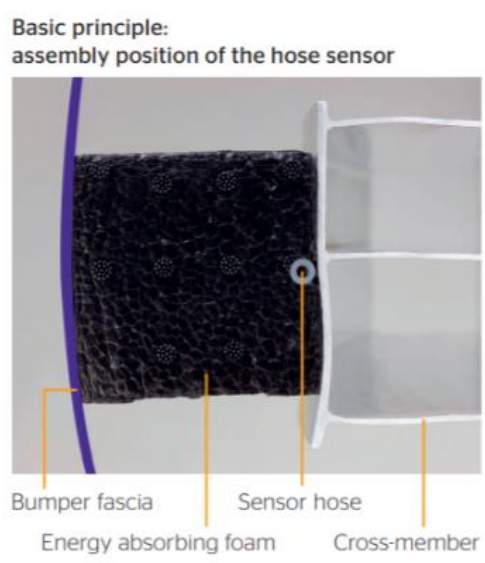

Fig5: Pressure sensor location in Bumper

Pedestrian protection is gaining more and more attention due to new legislation requirements and tougher consumer ratings. This is leading to the introduction of pedestrian protection systems, like active hood lifting. For triggering these actuators a reliable sensing system is required. Within $10-15$ milliseconds of an impact, the active hood of the vehicle or window airbag is triggered. This reduces the risk of death or severe injury to the pedestrian from hitting the hood and underlying engine block.

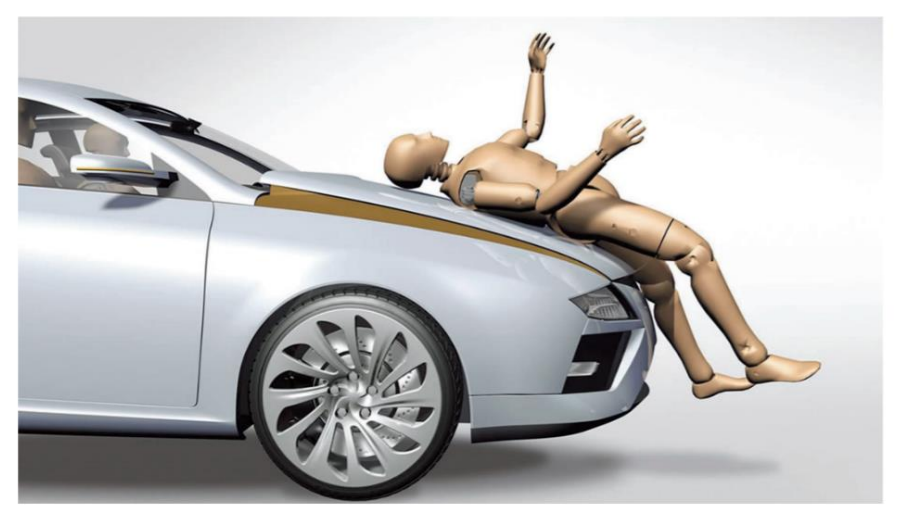

Fig5: Pressure-based pedestrian protection 


\section{3: Pedestrian sensing \& Detection}

Based on research article, a new working principle in car technology today is the pedestrian sensing and protection system. This system will be able to automatically detect when a person is walking in front of your vehicle within a close distance. For example, let's say you're driving through an intersection and a person is walking across the street in front of you. If you aren't paying attention to the road or if you turn your head away for just a few seconds, then you might not see them walking. This could result in you hitting them with your vehicle. However, with the pedestrian sensing and protection system, the car's sensors will detect that the person is close by and then automatically apply the emergency brake to stop the vehicle. The driver does not have to do anything.

1. Sensors - The car will know when someone is walking in front of your vehicle. If they are less than 10 feet away, the emergency brake will be applied to stop the vehicle from hitting them.

2. Video Camera - Some versions of this system will utilize video camera technology to detect a person in front of your vehicle. It will be able to recognize the distance and movement of the person instantly and then apply the brake when the vehicle gets too close.

3. Automatic Braking - Up until now, you would have to slam on your brakes when you notice someone passing in front of your car. If your reaction time isn't fast enough, you will still end up hitting the person anyway. The automatic braking of this protection system will ensure that the brakes are applied at the earliest time possible in order to avoid hitting the person

\section{Pedestrian detection}

The pedestrian detection uses advanced sensors to detect human movements; some versions may urgently apply the brakes if the driver fails to respond. The system alerts the driver or automatically brakes if there is a pedestrian in the path between a certain speed range-generally around 25 $\mathrm{mph}$. The advanced sensors used to detect human movements ahead and alert the driver. Some may automatically apply the brakes.

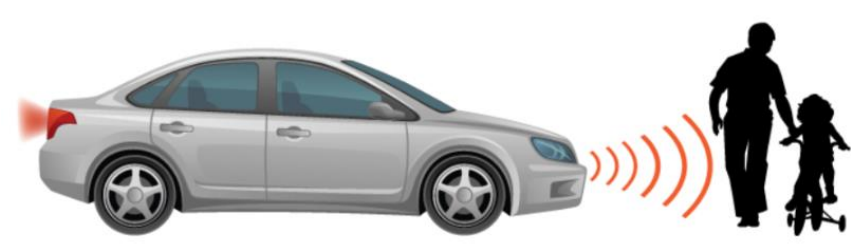

Fig6: Pedestrian detection approach

\section{Technology behind the Pedestrian detection technology:}

Stereoscopic cameras mounted behind the rear-view mirror and radar have become effective at detecting the more subtle movements of people.

These systems are more effective at slower speeds. Pedestrian Detection may not always be able to help avoid a collision, but this feature can help reduce the speed enough to make the impact more survivable. As research progresses, infrared technology is being added to improve performance, especially at night.

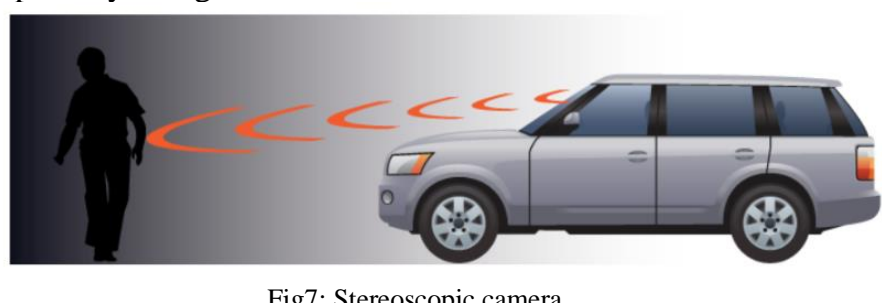

Fig7: Stereoscopic camera

\section{4: Video based approach}

Based on Alberto Broggi, University of Pavia, Italy review that video sensors are a natural choice for detecting people. Texture information at a fine angular resolution enables quite discriminative pattern recognition techniques. The human visual-perception system is perhaps the best example of how well such sensors might perform, if we add the appropriate processing. Besides, video cameras are cheap, and because they do not emit any signals, they raise no issues regarding interference with the environment. Considerable computer vision research deals with "looking at people." What makes pedestrian recognition applications on vehicles particularly challenging is the moving camera, the wide range of possible pedestrian

\section{5: Active Sensor approach}

Active sensors do not directly provide depth information; stereo vision derives depth by establishing feature correspondence and performing triangulation. On the other hand, active sensors measure distances directly

\section{6: Radar based approach}

Some commercial vehicles already employ radar for adaptive cruise control (for example, the Distronic System on Mercedes Benz S-Class cars). For near-distance applications, such as pedestrian detection, ongoing investigations focus on 24-GHz radar technology.14 Radar-based systems can enhance object localization by placing multiple sensors on the vehicle's relevant parts and applying triangulation-based techniques. They can classify objects - that is, distinguish pedestrians from other objects such as cars and trees-by examining the power spectral-density plot of the reflected signals. In this context, we consider an object's spectral content and reflectivity. Objects with smaller spatial extents, such as pedestrians, have narrower peaks in the plot than, say, cars. The material properties of the object's surface determine the strength of reflected radar signals. Vehicles metallic parts reflect much better than human tissue, by at least an order of magnitude. Human tissue, in turn, reflects much better than non-conductive materials, such as the wood in trees.

\section{7: Laser}

The main appeal of eye-safe laser range finders lies in their fast, precise depth measurement and their large field of view. For example, Martin Kunert, Ulrich Lages, and I describe a laser range finder that has a depth accuracy of $+/-5 \mathrm{~cm}$ and a range of $40 \mathrm{~m}$ for objects with at least 5 percent reflectivity (this includes most, if not all, relevant targets). Furthermore, 
its horizontal scans cover a 180-degree field of view in increments of 0.5 degree at $20 \mathrm{~Hz}$, making the sensor especially suitable to cover the area just in front of the vehicle.

\section{8: Lidar}

LiDAR uses scanning lasers to measure distances to surfaces, producing a three-dimensional map of detailed shapes. LiDAR is capable of object detection in low/no-light conditions, but like VLC, is unreliable in adverse weather and when road surfaces are wet or reflective. LiDAR is potentially useful for medium- and long-range detection, but is typically deployed as a single unit on a vehicle's rooftop, with its "view" of the ground surrounding the vehicle obstructed by the vehicle itself, hindering detection at very close range.

\section{SUMMARY FROM REFERENCES SURVEY}

Sensors' abilities to detect pedestrians in advance of fatal collisions vary from $<30 \%$ to $>90 \%$ of fatalities. Combining sensor technologies offers the greatest potential for eliminating fatalities, but may be unrealistically expensive. Furthermore, whereas initial deployment of automated vehicles will likely be restricted to freeways and select urban areas, non-freeway streets and rural settings account for a substantial share of pedestrian fatalities.

\section{CONCLUSIONS}

Although technologies are being developed for passenger vehicles to successfully detect pedestrians in advance of most fatal collisions, the current costs and operating conditions of those technologies substantially decrease the potential for passenger vehicles to radically reduce pedestrian fatalities in the short term.

\section{REFERENCES}

[1] Continental Automotive

[2] Bosch Automotive

[3] pSAT - Pressure Satellite which is trademark of Continental Automotive.

[4] gSAT -Acceleration Satellite which is Trademark of Continental Automotive. 